\title{
A Journey to Zero: Reduction of Post-Operative Cesarean Surgical Site Infections over a Five-Year Period
}

\author{
Evelyn Hickson, Jeanette Harris, ${ }^{2}$ and David Brett ${ }^{3}$
}

\begin{abstract}
Background: Surgical site infections (SSI) are a substantial concern for cesarean deliveries in which a surgical site complication is most unwelcome for a mother with a new infant. Steps taken pre- and post-operatively to reduce the number of complications may be of substantial benefit clinically, economically, and psychologically. Methods: A risk-based approach to incision management was developed and implemented for all cesarean deliveries at our institution. A number of incremental interventions for low-risk and high-risk patients including pre-operative skin preparations, standardized pre- and post-operative protocols, post-operative nanocrystalline silver antimicrobial barrier dressings, and incisional negative pressure wound therapy (NPWT) were implemented sequentially over a 5-y period. A systematic clinical chart review of 4,942 patients spanning all cesarean deliveries between 20072012 was performed to determine what effects the interventions had on the rate of SSI for cesarean deliveries.

Results: The percentage of SSI was reduced from $2.13 \%$ (2007) to $0.10 \%(2012)(p<0.0001)$. There were no substantial changes in the patient population risk factors over this time. As a result of the changes in incision management practice, a total of 92 cesarean post-operative SSIs were avoided: A total cost saving of nearly $\$ 5,000,000$. Conclusion: Applying a clinical algorithm for assessing the risk of surgical site complication and making recommendations on pre-operative and post-operative incision management can result in a substantial and sustainable reduction in cesarean SSI.
\end{abstract}

$\mathbf{T}$ HIRTY-TWO PERCENT of all deliveries in the United States were by cesarean section in 2007 with an estimated $48 \%$ of women ages $40-54$ giving birth via cesarean [1]. Although in recent years the rate of cesarean births has stabilized, cesarean delivery remains the most common operating room procedure in U.S. hospitals. Although most mothers recover uneventfully from a cesarean birth, a postoperative surgical site complication in the incision line can be devastating to a mother with a young infant trying to convalesce from the cesarean and at the same time care for the baby and frequently additional children.

In 2007 Multicare Health Systems (MHS) at Tacoma General (TG)-Washington State, identified as unacceptable the level of $2.13 \%$ cesarean surgical site infection (SSI) complications $(24 / 1,125)$ in our patient population. The National Healthcare Safety Network (NHSN) report notes a SSI rate for cesarean incisions of $1.46 \%$ across most patients in the lowest (National Nosocomial Infection Surveillance [NNIS]) risk index group (0), but in higher risk patients where the NNIS risk index is 2 or 3, the rate is $3.82 \%$ [2]. Although the incidence at our institution was in line with the NHSN report, taking the position that in our patient population any surgical site complication that could be avoided should be avoided, our response was the implementation of a risk-based approach to pre-operative, intra-operative, and post-operative incision management using a range of different interventions to bring the incidence to as low a level as possible. This report describes our review of progress toward a goal of a zero incidence of SSI in cesarean patients in all risk groups over the ensuing 5-y period.

\section{Patients and Methods}

Multicare Health Systems at Tacoma General (TG) is an inner city women's and newborn facility in the United States performing approximately 85 cesarean deliveries per month. In 2007 an incidence of SSI (2.13\%) across all cesarean incisions was recorded and it was hypothesized that by implementing a

\footnotetext{
${ }^{1}$ Perinatal Clinical Nurse Specialist, ${ }^{2}$ Surgical Specialist Infection Prevention, MultiCare Health System, Puyallup, Washington.

${ }^{3}$ Smith and Nephew Wound Management Division, St. Petersburg, Florida.
} 


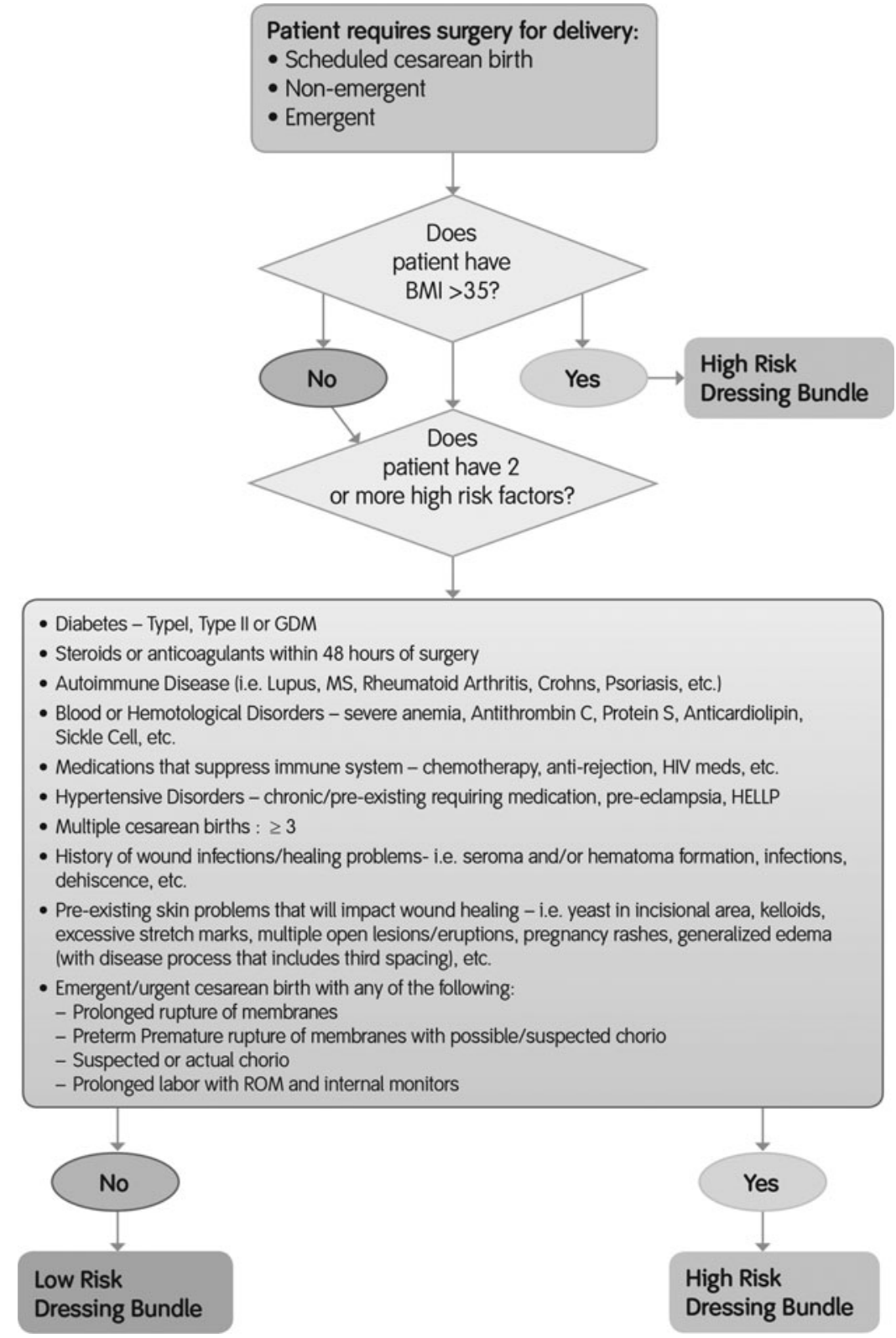

FIG. 1. Algorithm used to determine risk category (high or low) of a given patient and appropriate post-operative dressing bundle. $\mathrm{BMI}=$ body mass index; GDM=gestational diabetes mellitus; $\mathrm{MS}=$ multiple sclerosis; HIV=human immunodeficiency virus; HELLP = a form of severe preeclampsia, a rare but serious condition that occurs during pregnancy or just after delivery; chorio = chorioamnionitis, an inflammation of the fetal membranes due to a bacterial infection; ROM=rupture of membranes $(\mathrm{ROM})$ or amniorrhexis; $\mathrm{SSI}=$ surgical site infection.

number of quality improvement initiatives to pre- and postoperative care, that number could be reduced. Multiple factors were identified using LEAN principles (www.lean.org/whatslean/ principles.cfm). The LEAN paradigm is a manufacturing process approach that seeks to identify cause of waste and inefficiency in a process that results in the "customer" (in this case the patient) not receiving what they want (in this case "a cesarean section without wound healing complications"). Several factors contributing to SSI rates were identified and as a result a revised protocol was put in place. Patients were taught hygiene and basic infection prevention strategies. Hair was removed via clipping rather than shaving. Patients were asked not to wear makeup or jewelry, which could harbor bacteria. Hair was removed from the perineum and pre-operative showering became the norm. Chlorhexidine gluconate $(\mathrm{CHG})$ wipes rather than povidone-iodine (PVP-I) were used to prep the incision area [3]. Intra-operatively, extra care was taken when removing drapes and the use of sutures rather than staples on fat layers $>2 \mathrm{~cm}$ were introduced [4]. Post-operative wound interventions were also revised: Dressings were changed on day three unless required because of saturation. Staples were removed on day 7-10, not at discharge. As the protocol evolved, patients were separated into either a high-risk or low-risk category. Principal risk factors for SSI wound healing complications in cesarean deliveries were identified and an algorithm was drawn up to categorize all women into either high- or low-risk [5]. Women 
Table 1. Participating Patients and Decrease in Surgical Site Infections during the Study Period

\begin{tabular}{|c|c|c|c|c|c|c|}
\hline Year & 2007 & 2008 & 2009 & 2010 & 2011 & 2012 \\
\hline Patients with SSI & 24 & 21 & 17 & 7 & 6 & 1 \\
\hline Total cesarean section & 1,125 & 1,008 & 998 & 988 & 984 & 964 \\
\hline$\%$ SSI & 2.13 & 2.08 & 1.70 & 0.71 & 0.61 & $0.10 *$ \\
\hline Decrease in 2007 & & $2.3 \%$ & $20.2 \%$ & $66.7 \%$ & $71.4 \%$ & $95.3 \%$ \\
\hline
\end{tabular}

*Absolute decrease 2007-2012: 2.03\%

$\mathrm{CI}=$ confidence interval: $1.14 \%-3.06 \%$; Fisher exact test $\mathrm{p}<0.0001$.

$\mathrm{SSI}=$ surgical site infection .

with a body-mass index (BMI) in excess of $35 \mathrm{~kg} / \mathrm{m}^{2}$ were placed in the higher risk category. Additionally designated high risks were women with any two of a number of different factors such as diabetes mellitus, smoking, immunosuppression, and emergency cesarean (Fig. 1). The low-risk post-operative dressing bundle (introduced in 2011) consisted of a film-forming skin preparation (pre-incision), topical skin- adhesive (2-octyl cyanoacrylate), nanocrystalline silver anti-microbial barrier dressing, see-through barrier dressing (non-medicated), and film-forming skin preparation (used around edges of dressing to seal). Prior to 2011 standard post-operative dressings were used (soft cloth adhesive dressings and absorbent cotton gauze dressings). The high-risk dressing bundle (introduced in 2012) consisted of a film-forming skin preparation (pre-incision), topical skin adhesive (2-octyl cyanoacrylate), nanocrystalline silver anti-microbial barrier dressing, film-forming skin preparation, single-use negative pressure wound therapy system, and film-forming skin preparation (used around edges of dressing to seal). Traditional negative pressure wound therapy (tNPWT) was used on high-risk patients (2008-2011) but only occasionally. Only 30\% of high-risk patients received tNPWT. In 2012 tNPWT was replaced with the single-use NPWT system in the formal high-risk dressing bundle implemented in 2012. All patients $(n=4,942)$ who underwent a cesarean delivery from 2007-2012 were included in the final analysis. Data collection was achieved by reviewing patient records, which contained all follow-up information as well as initial diagnosis. Surgical site infection rates were recorded at 5-7 d after surgery, at 2-3 wks after surgery, and 6 wks after surgery. The rate of SSI was determined (using NHSN definitions) monthly over the course of pre-intervention and intervention and then also followed throughout the study to determine adherence to the intervention. Data were mined via MedMined ${ }^{\circledR}$ (CareFusion, San Diego, CA) allowing capture of all positive cultures throughout the system including all inpatient, urgent care, and emergency departments plus $250+$ outpatient clinics. Any positive cultures generate a nosocomial infection marker that was then investigated for postoperative infection. The institutional board deemed that this retrospective review did not require research ethics approval. Fisher exact test was used to compute $\mathrm{p}$ values for differences in proportions and corresponding asymptotic $95 \%$ confidence intervals were generated.

\section{Results}

Over the $5-y$ period $33.77 \%$ of the patients had no risk factors, whereas $66.23 \%$ had a BMI of $>35$ and/or at least two of the risk factors. The mean BMI of patients over the 5-y period was $35( \pm 7) \mathrm{kg} / \mathrm{m}^{2}$ and the mean age was $28( \pm 6) \mathrm{y}$. Table 1 shows the incidence of SSI for each year of the analysis. There was little change in rates of SSI in 2008 $(2.08 \% ; 21 / 1008)$ and $2009(1.7 \% ; 17 / 998)$. The greatest change came in 2010, when SSI rates decreased to $0.71 \%$ (7/988). During this time frame intervention such as shaving (rather than clipping), patients not wearing makeup or jewelry, and shaving the perineum were implemented. Chlorhexidine gluconate wipes and pre-operative showering was also implemented during this time period. The current lowrisk dressing bundle was initiated and utilized throughout 2011 where rates remained broadly unchanged at $0.61 \%$ (6/984). The high-risk dressing bundle was revised in 2012 to treatment with a single-use NPWT system, $\left(\mathrm{PICO}^{\mathrm{TM}}\right.$, Smith \& Nephew Hull, London, UK) for prophylactic NPWT on all high-risk patients [6]. The PICO Single Use Negative Pressure Wound Therapy System is a canister-free, portable, disposable device consisting of a pump and sterile dressings. The PICO pump maintains NPWT of $80 \mathrm{~mm} \mathrm{Hg}$ at the wound surface. Exudate is managed by the dressing through a combination of absorption and evaporation of moisture through the outer film. The incidence of SSI in 2012 was $0.10 \%(1 / 964)$. Application of NPWT to a closed incision has been shown to reduce incidence of infection and dehiscence [7,8]. Although none of the year by year changes is significant, the overall change in incidence of SSI from $2.13 \%$ to $0.1 \%$ is a statistically significant reduction $(\mathrm{p}<0.0001)$.

\section{Cost Analysis}

The initial review of practice and materials used in 2007 revealed the cost for managing a low-risk patient was $\$ 32.94$. The cost of incisional bolstering with traditional NPWT required by a high-risk patient is $\$ 348.62$ (3 d of tNPWT). The proposed changes for incisional closure, dressing, and postpartum incisional management for a low-risk patient resulted in a cost of $\$ 42.69$ (increase of $\$ 9.75$ per patient) compared with 2007 costs. However, a high-risk patient with incisional bolstering with the single-use negative pressure device is $\$ 245.30$ (7 d of NPWT). A savings of $\$ 103.32$ per patient compared with tNPWT. Ninety-two cesarean SSIs have been avoided since implementing the bundle, yielding an approximate cost savings of nearly $\$ 5,000,000$ (based upon a historic average of $\$ 50,000$ per readmission).

\section{Discussion}

Increasing attention is being paid to the costs and consequences of post-operative surgical site complications after cesarean delivery [9]. Analysis of risk factors reveals that high BMI plays a substantial role with studies finding complications as high as $20 \%$ in patients in whom BMI exceeds 
$35 \mathrm{~kg} / \mathrm{m}^{2}$ within an overall population incidence of $12 \%$ SSI [10]. In the patient population served by Multicare Health Systems, more than two-thirds of patients had BMI above 35 and although our identified rates of SSI were below those in other reports we instigated a comprehensive improvement program with pre-operative and post-operative changes in practice that yielded an absolute reduction in caesarian section SSI from $2.13 \%$ to $0.1 \%$; ( $<<0.0001)$. However, it is not possible to identify individual interventions that are uniquely responsible for SSI incidence reduction from a program that included alterations in patient hygiene, surgical site preparation, antimicrobial post-operative dressings, and incisional NPWT.

Although not statistically significant, the greatest decrease in SSI came in 2010 where the intervention focused on better patient hygiene pre- and post-operatively. Also, $\mathrm{CHG}$ replaced PVP-I for preparation of the incision area. A recent retrospective report compared two groups of patients with cesarean delivery incisions covered with gauze pads or an anti-microbial silver dressing [11]. No differences were observed, although the study was small and non-randomized. Other reports have suggested post-operative silver dressings may reduce incidence of SSI in different wound types [12,13]. NPWT applied to closed incisions using full-sized durable NPWT pumps has been reported to reduce the incidence of post-cesarean SSI [14] although this study examined a morbidly obese group in which BMI was in excess of $45 \mathrm{~kg} / \mathrm{m}^{2}$. The evidence and mechanisms for incisional NPWT has recently been reviewed systematically [15]. Interestingly, in our institution the introduction of a number of new postoperative practices was able to show reduction in SSI, even when rates were rather modest and in line with data averaged over many U.S. facilities [2].

\section{Acknowledgments}

E.H. and J.H. are employees of Multicare Health Systems, Inc. No commercial funding was received for initiating the changes in post-operative management or this analysis. Wound care consultation, data analysis, and assistance in manuscript development were provided by Smith \& Nephew, Inc. D.B. is an employee and a shareholder of Smith \& Nephew, Inc. We thank Robin Martin (Smith \& Nephew) for help in revising the article.

\section{Author Disclosure Statement}

E.H. has acted as a consultant for a fee and been paid or sponsored by the organization (Smith \& Nephew) to speak to professional or lay groups. J.H. has been paid or sponsored by the organization (Smith \& Nephew) to speak to professional or lay groups. D.B. is an employee and a shareholder of Smith \& Nephew, Inc.

\section{References}

1. Menacker F, Hamilton BE. Recent trends in cesarean delivery in the United States. NCHS Data Brief 2010; March:1-8.
2. Edwards JR, Peterson KD, Mu Y, et al. National Healthcare Safety Network (NHSN) report: Data summary for 2006 through 2008, issued December 2009. Am J Infect Control 2009;37:783-805.

3. Dumville JC, McFarlane E, Edwards P, et al. Preoperative skin antiseptics for preventing surgical wound infections after clean surgery. Cochrane Database Syst Rev 2013;3: $1-66$.

4. Basha SL, Rochon ML, Quiñones JN, et al. Randomized controlled trial of wound complication rates of subcuticular suture vs staples for skin closure at cesarean delivery. Am J Obstet Gynecol 2010;203:285.e1-8.

5. Sarsam SE, Elliott JP, Lam GK. From cesarean delivery. Wounds 2005;60:462-473.

6. Hudson DA, Adams KG, Van Huyssteen A, et al. Simplified negative pressure wound therapy: Clinical evaluation of an ultraportable, no-canister system. Int Wound J 2013;1:1-7.

7. Stannard JP, Robinson JT, Ratcliffe Anderson E, et al. Negative pressure wound therapy to treat hematomas and surgical incisions following high-energy trauma. J Trauma 2006;60:1301-1306.

8. Gomoll AH, Lin A, Harris MB. Incisional vacuum-assisted closure therapy. J Orthopaed Trauma 2006;20:705-709.

9. Olsen MA, Butler AM, Willers DW, et al. Attributable costs of surgical site infection and endometritis after low transverse cesarean delivery. Infect Control Hosp Epidemiol 2010;31:276-282.

10. Wloch C, Wilson J, Lamagni $\mathrm{T}$, et al. Risk factors for surgical site infection following caesarean section in England: Results from a multicentre cohort study. BJOG 2012; 119:1324-1333.

11. Connery SA, Downes KL, Young C. A retrospective study evaluating silver-impregnated dressings on cesarean wound healing. Adv Skin Wound Care 2012;25:414-419.

12. Childress BB, Berceli SA, Nelson PR, et al. Impact of an absorbent silver-eluting dressing system on lower extremity revascularization wound complications. Ann Vasc Surg 2007; 21:598-602.

13. Krieger BR, Davis DM, Sanchez JE, et al. The use of silver nylon in preventing surgical site infections following colon and rectal surgery. Dis Colon Rectum 2011;54: 1014-1019.

14. Mark KS, Alger L, Terplan M. Incisional negative pressure therapy to prevent wound complications following cesarean section in morbidly obese women: A pilot study. Surg Innov 2013;21:1-5.

15. Karlakki S, Brem M, Giannini S, et al. Negative pressure wound therapy for management of the surgical incision in orthopaedic surgery: A review of evidence and mechanisms for an emerging indication. Bone Joint Res 2013;2:276-284.

Address correspondence to: David Brett 920 Lake Carillon Dr. St. Petersburg, FL 33716

E-mail: dave.brett@smith-nephew.com 